\title{
IMPLEMENTING AIR-POLLUTION AND HEALTH-DAMAGE COSTS IN URBAN MULTI-ENERGY SYSTEMS MODELLING
}

\author{
MARCO RAVINA ${ }^{1}$, EDOARDO PATTI ${ }^{2}$, LORENZO BOTTACCIOLI $^{2}$, DEBORAH PANEPINTO $^{1}$, \\ ANDREA ACQUAVIVA ${ }^{3} \&$ MARIA CHIARA ZANETTI ${ }^{1}$ \\ ${ }^{1}$ Department of Engineering for Environment, Land and Infrastructures, Politecnico di Torino, Italy \\ ${ }^{2}$ Department of Control and Computer Engineering, Politecnico di Torino, Italy \\ ${ }^{3}$ Interuniversity Department of Regional and Urban Studies and Planning, Politecnico di Torino, Italy
}

\begin{abstract}
The growing global urbanization rate implies that the sustainability challenges are increasingly concentrated in cities. At today, around $75 \%$ of global energy is consumed in urban areas, so efforts must be addressed to transform existing urban energy systems into more sustainable systems. In this perspective, a key aspect to evolve toward a cleaner and affordable energy system is the development of Multi-Energy Systems (MES) modelling, whereby heat, electricity, fuels, transport, and other energy carriers closely interact with each other at various scales. MES can optimize technical, economic and environmental performance with respect to "traditional" independent energy systems, at both the operational and the planning stage. This paper presents a development of the existing MESsi modelling platform, consisting in the implementation of a model estimating the impacts on air quality and human health. MESsi is a novel distributed infrastructure for modelling and co-simulating Multi-EnergySystems. It exploits modern software design patterns (i.e. microservices) to guarantee scalability, extendibility and easy maintenance of the system. Thus, MESsi is flexible in modelling and cosimulating different energy flows in a single solution made of different interoperable modules that can be deployed in a plug-and-play fashion. The module to be implemented in MESsi infrastructure is the DIATI integrated dispersion and externalities model (DIDEM). The DIDEM model is based on the impact pathway approach, linking the simulation of pollutants dispersion to the concentrationexposure-response functions provided by latest WHO recommendations. An overview of the potential integration steps in the modelling infrastructure is described in this paper. A discussion on possible application scenarios that have different spatio-temporal resolutions is also reported. The integration of DIDEM model in MESsi platform allows the inter-connection of a detailed impact assessment to a high-level energy system simulation.
\end{abstract}

Keywords: multi-energy systems, modelling, air pollution, impact pathway approach, health effects.

\section{INTRODUCTION}

The energy sector is a source of economic and social progress, but it is also the main responsible of air pollution resulting from human activity, mainly from the conversion of fossil fuels and other energy sources. Worldwide energy and environmental policies are addressed to minimize energy consumption and encourage the use of Renewable Energy Sources (RES). Moreover, novel ICT (Information Communication Technology) solutions can increase the demand flexibility by managing the uncertain production of RES and by optimizing the energy consumption in cities. In this context, Multi-Energy-Systems (MES) modelling represents an essential tool in support of energy and environmental decisionmaking processes [1]. MES modelling tools implement electricity, heat, cooling, fuels and transport networks, simulating their interaction at various levels (within a district, or a city, or at a country level) and exploiting novel ICT solutions, such as cyber-physical-systems, Internet-of-Things (IoT), cloud computing and cognitive computing.

As highlighted by previous research studies [1], [2], an in-depth simulation and analysis of MES is required to increase the flexibility of energy systems by integrating different 
resources for both electric and thermal energy. Furthermore, ICT and MES offer valid options to foster novel services for smart energy management. For example, they can foster events of Demand Response (DR) and Demand Side Management (DSM) by integrating buildings equipped with heat pumps, CHP (Combined Heat Power) or HVAC (Heating, Ventilation and Air Conditioning) systems [3]. In [3]-[6], authors present a complete overview of literature tools and models for MES analysis.

A possible and future extension of the scope of MES modelling tools is represented by the implementation of environmental and health risk aspects. In fact, energy scenarios must be assessed not only in terms of energy balance, but also in terms of i) environmental impacts and ii) air-pollutant induced health effects, and the associated definition of external costs [7]. Impacts of air pollution may be observed both at the local and the global scale. At the global scale, greenhouse gas emission generates impacts on the climate system, bringing changes in temperature and weather patterns [8], crop loss, and increased incidence of certain diseases. At the local scale, the emission of macro and micro-pollutants generates impacts on humans and ecosystems [9]. These unexpected or unwanted impacts are defined as externalities, i.e. those effects on the wellbeing of an unrelated group or individual outside the market mechanism that controls the price of energy. External costs, or damage costs, are the monetary value of externalities. Considering health effects of air pollution, the currently applied methodology is the impact pathway approach (IPA) [10]-[12]. IPA is considered a valuable application for energy systems planning and management. Modelling tools have been widely used to implement the estimation of externalities associated to air pollution [13]. Their application fosters the screening and detection of the key-factors that affect, directly or indirectly, the decision process.

In this paper, the potential implementation of an integrated IPA model in an existing MES simulation platform is described. The considered distributed infrastructure is called MESsi, conceived at Department of Control and Computer Engineering of Turin Polytechnic, Italy [14]. MESsi was designed to be applied in an urban context by exploiting novel ICT solutions, such as cyber-physical-systems, Internet-of-Things (IoT), cloud computing and cognitive computing.

The integrated environmental - externalities tool to be implemented is called DIDEM (DIATI Dispersion and Externalities Model), developed at the Department of Engineering for Environment, Land and Infrastructures (DIATI) of Turin Polytechnic, Italy. The DIDEM model was designed to perform an analysis of external health impacts and costs by comparing two or more operating scenarios. DIDEM model was conceived to work with fine spatial resolution and a local geographic scope (cities, metropolis or similar areas).

Considering the architecture and scope of both systems, DIDEM is considered a potential valid complementary tool for MESsi. MESsi combines together different technologies and heterogeneous information to model the energy flows and to simulate the impact of novel control strategies in cities and distribution networks. It also can exploit information coming in (near-) real-time from Internet connected devices installed across the city. Furthermore, MESsi provides features to simulate how such novel policies affect the energy marketplace and to analyse the effects and/or limitations of regulatory frameworks. On these premises, MESsi is an infrastructure for simulations as a service that can be used by different stakeholders to build and analyse new energy scenarios for short- and long-term planning activities. Similarly, the DIDEM model was conceived and applied to compare the compatibility of different industrial options such as energy scenarios, but also various aspects, as local policy planning or forecasting scenarios [16].

Given these premises, even if coming from different research fields, MESsi and DIDEM may be considered part of a common objective. This paper aims at: i) analysing the structure 
of MESsi and DIDEM; ii) detect and describe the potential point of connections of the systems; iii) give an overview on the procedural steps required for the implementation of DIDEM in MESsi, and iv) report some consideration on the possible application of an updated MESsi platform, with implemented environmental and externalities estimation. In this perspective, the case study of Turin currently developed by the Energy Center Lab (ECLAB) research group at Turin Polytechnic is described. In this research activity, MESsi constitutes the structural basis of the modelling framework. In this section, a particular focus is addressed to the multi-scale approach, which represents a key-aspect in energy and environmental modelling at the urban scale.

This paper is structured as follows: in section 2, MESsi platform is described; in section 3, DIDEM model is described; in section 4 an analysis of the implementation of DIDEM in MESsi is reported; in section 5 the concluding remarks are reported.

\section{THE MESSI PLATFORM}

In this section, we briefly present MESsi [14], which is a distributed online infrastructure for near-real-time modelling and co-simulation of Multi-Energy-Systems in urban areas. It implements features for different spatio-temporal resolutions spanning i) from single dwelling to districts and cities (space resolution), and ii) from microseconds to years (time resolution). For example, MESsi can simulate either the operational status of distribution networks or long-term planning and refurbishment activities in cities.

As shown in Fig. 1, we identified 5 layers:

- The Environmental layer integrates heterogeneous data-sources that describe the urban area as whole.

- Geographical Information System (GIS) provides i) georeferenced information about the different entities in cities such as devices, buildings and pipelines; ii) cartographies cadastral maps and iii) Digital Elevation Models.

- Building Information Models (BIM) are parametric 3-Dimensional models providing both structural and semantical descriptions of buildings in cities.

- System Information Models (SIM) describe size and structure of energy distribution networks.

- Weather Data are georeferenced information collected by weather stations in cities that are made available through third-party services (e.g. Weather Underground).

- Census data are statistical information on different characteristics and behaviours of citizens.
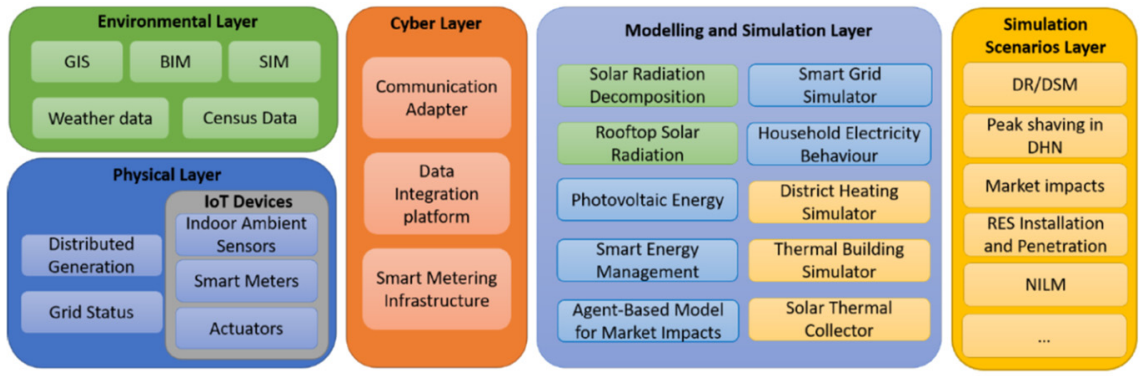

Figure 1: Representation of the actual architecture of MESsi modelling platform. 
- The Physical layer integrates heterogeneous Internet-connected devices that send information in near-real-time, such as multi-vector measurements, status of distribution networks and/or actuation commands.

- The Cyber layer allows both synchronous and asynchronous bidirectional communication among the different actors in MESsi. The Communication Adapter and the Data Integration Platform integrate the heterogeneous Internet-connected devices in the Physical Layer and (third-party) software data-sources in the Environmental Layer, respectively. Smart Metering Infrastructure, such as Flexmeter [15], makes available historical data collected from real distribution networks and post-processed information output of its services.

- The Modelling and Simulation layer consists of various modules to simulate environmental conditions (green boxes); electrical energy (light blue boxes), for both production and consumption; and thermal energy (yellow boxes). These modules implement feature for multiscale spatio-temporal resolutions. The Solar Radiation Decomposition module [24] decomposes the Global Horizontal radiation into Direct Normal Incident radiation and Diffuse Horizontal Incident radiation by applying mathematical models in literature. The Rooftop Solar Radiation module [24] simulates incident solar radiation on rooftops in real-sky conditions. The Photovoltaic Energy module [24] estimates the energy production of PV systems with 15-minute resolution. The Agent-Based Model for Market Impacts module simulates the impact of renewable energy sources and novel control policies on the electrical marketplace. The Smart Grid Simulator module [15], [25] integrates RealTime Simulators (e.g. Opal-RT or RTDS) to evaluate the behaviour of power distribution networks in certain operational conditions. The Household Electricity Behaviour module reproduces realistic electrical consumptions in residential houses. Both District Heating Simulator [26] and Smart Energy Management [27] modules simulate novel control policies for energy optimization in heating and power distribution networks, respectively. The Thermal Building Simulator [28] provides tools to simulate and analyse the thermal behaviour of buildings. Finally, The Solar Thermal Collector simulates the behaviours of solar thermal panels.

- The Simulation Scenarios layer makes available to end-users a set of predefined tools and API (Application Programming Interface) to access raw or post-processed information from other modules and layers in MESsi. Thus, various scenarios can be built and run with different spatio-temporal resolutions according to stakeholders' requirements.

\section{THE DIDEM MODEL}

The DIDEM model was designed to calculate the environmental impacts and the external costs associated to the comparative analysis of emission scenarios. The term external costs refer to the marginal health damage costs, i.e. those costs generated by the effects on human health resulting from an extra unit of pollutant concentration. DIDEM model can be used to compare the compatibility of different industrial options, but also other aspects, such as local policy planning or forecasting scenarios. The following features are implemented:

- The integration of a detailed and advanced pollutant dispersion model (CALPUFF, [19]) with the calculation of health concentration-response functions (CRFs), implemented following the latest WHO recommendations [17], [18]; 
- The implementation of different confidence levels on CRFs data reported by the WHO, resulting in a precise estimation of uncertainty associated to the calculation of health effects;

- The implementation of updated monetary values of health effects introduced by the EU's Clean Air Policy Package.

The DIDEM model was conceived basing on the impact pathway approach (IPA) methodology (Fig. 2). The code was developed with MATLAB ${ }^{\circledR}$ software and conceived as a desktop (offline) tool. It was previously tested on case studies related to the operation of the district heating network of the Italian city of Turin [22], [23].

The user is allowed to input customized emission flows, including both point and area sources, as well as customized data of population exposure. For each scenario, the input to the model is the hourly emission flow of $\mathrm{NO}_{\mathrm{x}}$ (as equivalent $\mathrm{NO}_{2}$ ), $\mathrm{PM}_{2.5}$ and $\mathrm{PM}_{10}$. The DIDEM model organizes the hourly series in a compatible format to CALPUFF and executes CALPUFF. Once the CALPUFF simulation is terminated, the DIDEM model extracts and re-formats the output concentration grids and calculates the concentration differences. These latter are passed to the final module calculating the delta-external costs, which represents the core of the model.

The DIDEM model can be executed entirely by running a control script (didem.m); in alternative, each module can be run separately. Input and output from each module is reported in Fig. 3.

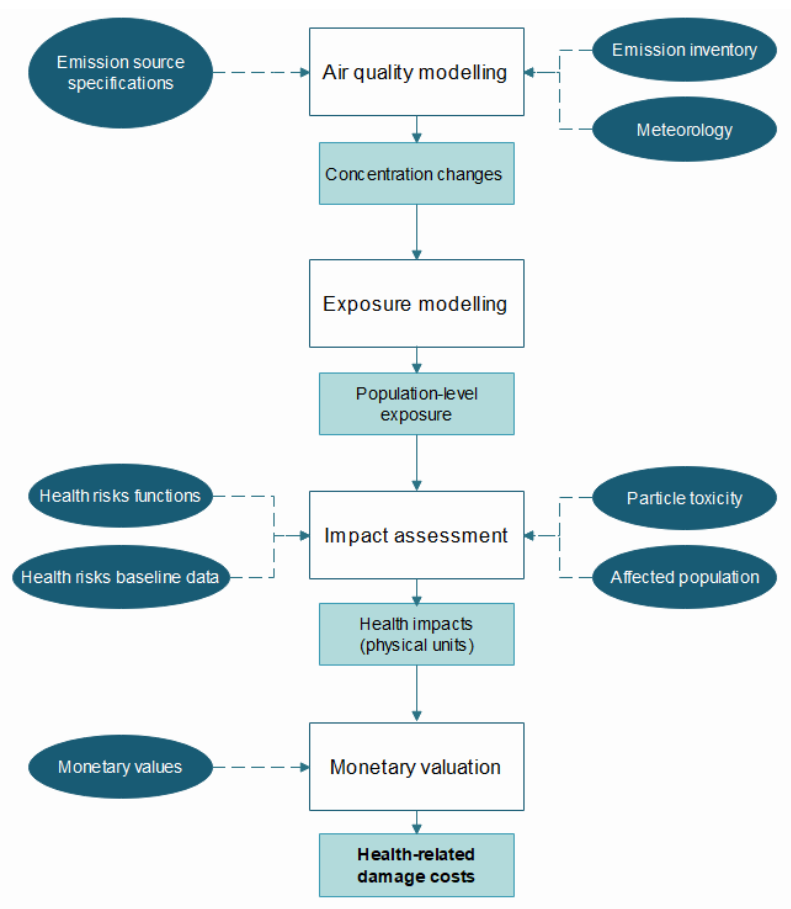

Figure 2: Schematic representation of the impact pathway approach. 
The final output provided by DIDEM is:

- Grids of concentration difference of $\mathrm{NO}_{\mathrm{x}}, \mathrm{PM}_{2.5}$ and $\mathrm{PM}_{10}$ over the modelling domain. Grids are provided in ASCII format, in order to be manageable with SURFER $^{\circledR}$ software [20] or GIS tools like QGIS [21].

- Table of five maximum and minimum concentration difference, with the related position in the spatial domain and the time of occurrence;

- Grids of distribution of delta external costs over the modelling domain;

- Tables reporting the total variation in external costs associated to the considered scenarios. The results reported herein are differentiated depending on the level of confidence of the input health effect/response pairs considered. An estimation of maximum and minimum range of uncertainty is also reported.

\section{IMPLEMENTATION OF DIDEM IN MESSI}

This section describes schematically the scheduled procedure for the implementation of DIDEM model into MESsi platform. This action represents part of the research activity currently performed by the Energy Center Lab (EC-LAB) research group at Turin Polytechnic, Italy. The following sections report i) an overview of the main technical and methodological steps defining the concept of the integrated platform and ii) a brief presentation of the research activity at EC-LAB and the case study of Turin city.

\subsection{Overview of the integration procedure}

In principle, the implementation of DIDEM in MESsi is addressed to i) share some status variables as an input and ii) use outputs coming from energy balance calculation to estimate the consequences on human health and the environment.

Referring to the multi-layered structure of MESsi, reported in Fig. 1, the integration procedure starts from the detection and analysis of the connection nodes commonly shared by DIDEM and MESsi. These connections are schematically reported in Fig. 3.

Considering the spatial scope and resolution, the common modular multi-scale approach makes MESsi and DIDEM highly compatible for the simulation of different scenarios, from the single building to the complete urban scale.

On the temporal scope and resolution, it must be pointed out that DIDEM is not presently designed for real-time simulations. Its application is thus limited to the scenarios evaluation against a baseline scenario, based on established time frames (e.g. one reference season or year).

Considered MESsi multi-layered structure, a number of actions and considerations may be defined by the analysis of single layers:

- Environmental layer. Information coming from the environmental layer represents an input as well as a target for DIDEM model. Weather and geophysical data are essential drivers for the calculation of the effects on air quality resulting from energy scenarios. These are processed and introduced in the pollutant dispersion model. Census data provide important information on the definition of emission sources (e.g. height of buildings), and (must of all), on population, which represents the final target of health effects and costs estimation with DIDEM. GIS-based data also represent a valid source of information for the characterization of emission sources. In addition, geo-localization results useful in representing simulation results. Finally, BIM modules may be connected to DIDEM in the view of a down-scaled 
scenario assessment (e.g. study and analysis of reference solutions and options at the building scale).

- Physical layer. In principle, a connection node to DIDEM must be arranged each time an emission source is present. The information passed to DIDEM will include: location of each source, geometric features of the sources and features of the conversion units (type, fuel etc.). Data coming from IoT devices (e.g. monitoring of background pollutants concentration) could be also used and implemented in DIDEM's dispersion module.

- Cyber layer. On the one hand, DIDEM exploits Web Services API to retrieve the needed inputs from the modules in both Environmental and Physical layers by exploiting the Data Integration Platform and the Communication adapters, respectively. On the second hand, DIDEM uses this layer to enable information exchange with other modules in the Modelling and simulation layer by exploiting their API.

- Modelling and simulation layer. The existing simulation models implemented in MESsi influence, directly or indirectly, the performance and results of DIDEM model. As for the Physical layer, a connection of each module to DIDEM must be arranged each a time an output from one or modules implies an emission of pollutants into the atmosphere. Considering the present configuration of MESsi, the thermal building simulator and the district heating simulator can provide emission data to DIDEM. These data must include the magnitude and the time variability of both energy flow and pollutants flow. In a perspective of a continuous progressive development and integration of the modelling platform, other components shall be implemented, such as emissions from traffic or industrial activities.

- Simulation scenarios layer. In the current assumed no-real time configuration, DIDEM can be considered passive with respect to the Simulation scenarios layer. This means that, in the first stage of implementation, DIDEM will only estimate the effects of a given scenario generated by MESsi's specific module. the results provided by DIDEM represent the effects of the application of a given scenario. In a future perspective, DIDEM structure may be modified to enable an inverse modelling of scenarios (e.g.: calculate an energy configuration starting by a result of DIDEM model, such as a pollutant reduction scheme).

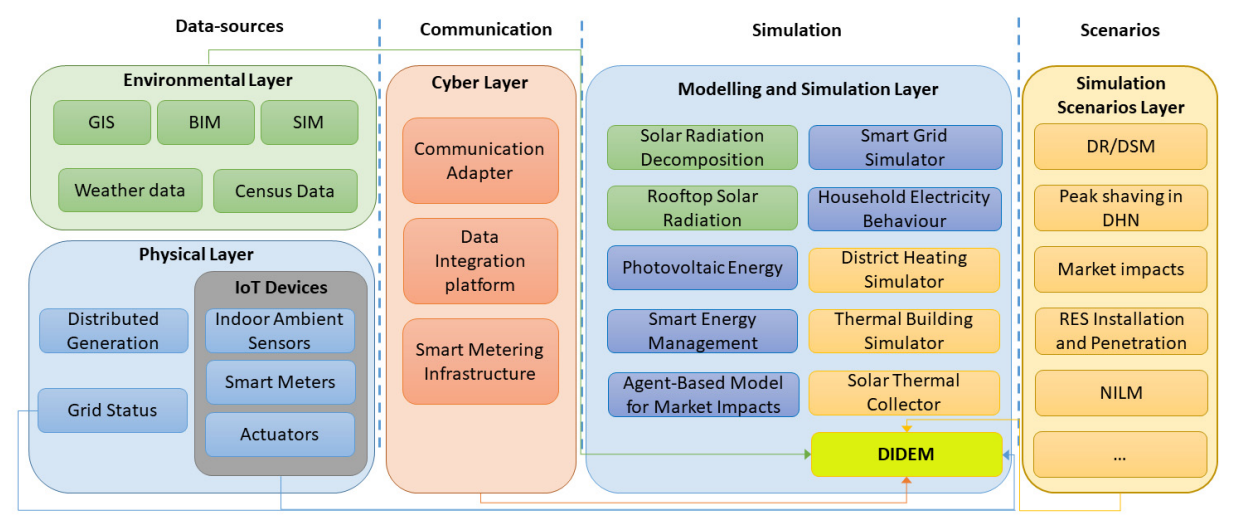

Figure 3: Connection of DIDEM to MESsi modelling platform. 


\subsection{The activity of EC-LAB and the case study of Turin, Italy}

The MESsi platform constitutes the structural and theoretical basis of the research activity on energy systems modelling currently carried on by the Energy Center Lab (EC-LAB) research group at Turin Polytechnic, Italy [29]. The Energy Center Lab is one of the interdepartmental centers of Turin Polytechnic. Its mission is evaluating the impacts on the energy system of introducing new technologies, infrastructures and policies, to develop energy transition scenarios.

The objective of the research at EC-LAB is the development of an energy scenario making (ESM) modelling platform, able to simulate complex energy systems and analyse different management scenarios and their effects on the social, economic and environmental spheres. MESsi model constitutes the starting point of the ESM platform. The ESM platform is currently being engineered and tested on a real case study, corresponding to the energy system of the Italian town of Turin.

The philosophy underlying the development of the ESM platform is founded on the MES concept, and is extended to include all those actors and layers that might directly or indirectly influence the behaviour of the energy system. These layers are:

- Technological layer (conversion, storage, distribution, transmission, final use);

- Environmental layer (effects and impacts on the environment);

- Informatics/ IoT layer (data access and analysis);

- Economic layer (energy markets, subsidies, competitiveness, demand analysis);

- Social layer (user behaviour, people involvement, health, safety).

Another element of innovation of the ESM platform is the adaptability to Hardware-inthe-loop (HIL) simulations, in connection to the experimental section of the EC-LAB. HIL simulations are important, as they allow real time connection of hardware technologies to the model to test their performance with a higher level of system integration.

The ESM platform project started with a first action consisting in the design and development of the platform and the definition of protocols for the integration of new modules. The scheduled tasks include: i) the realisation of the installation protocols of new modules into the platform; ii) the design of interoperability protocols between modules and third parts; iii) the realisation of solving algorithms for the scenarios making methodologies and iv) the design of a graphical user's interface (GUI). At the same time, the different models potentially suitable to be implemented in the ESM platform have been screened and evaluated.

The adjustment and application of the ESM platform to the energy system of Turin has the final objective of providing support to local policy makers for the definition and planning of efficient energy policies. The areas of intervention are several and involve the introduction or empowerment of technologies and services to improve energy efficiency, air quality and citizens well-being. In particular, the models will be tuned to evaluate the effects of an improved management of transmission grids, through the simulation of demand-side management (DSM) and virtual power plant (VPP) solutions. The operating scenarios will be constructed according to local and national energetic and environmental action plans.

In this context, reducing pollution through a peak shaving in District Heating Network (DHN) is reported as an example of DSM simulation scenario that exploits and combines together both MESsi Platform and DIDEM model. Fig. 4 reports the time diagram with the interaction between the involved modules of the platform. 


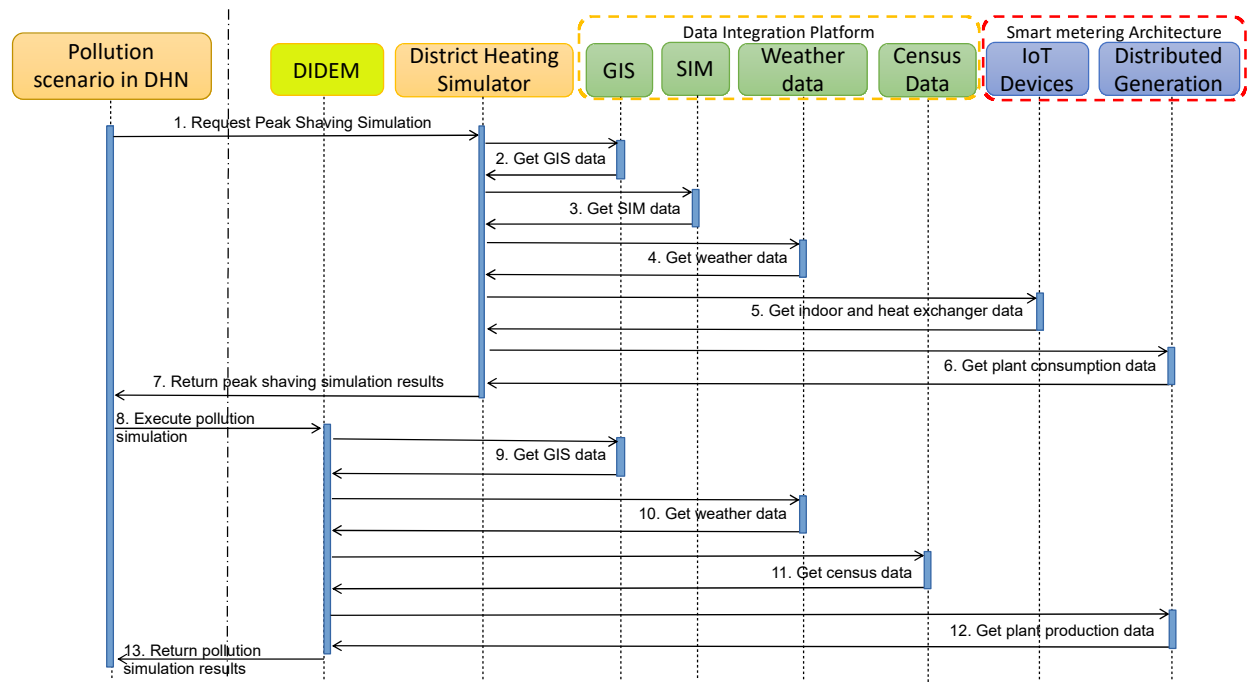

Figure 4: Time Diagram for Pollution reduction simulation due to Peak Shaving in DHN.

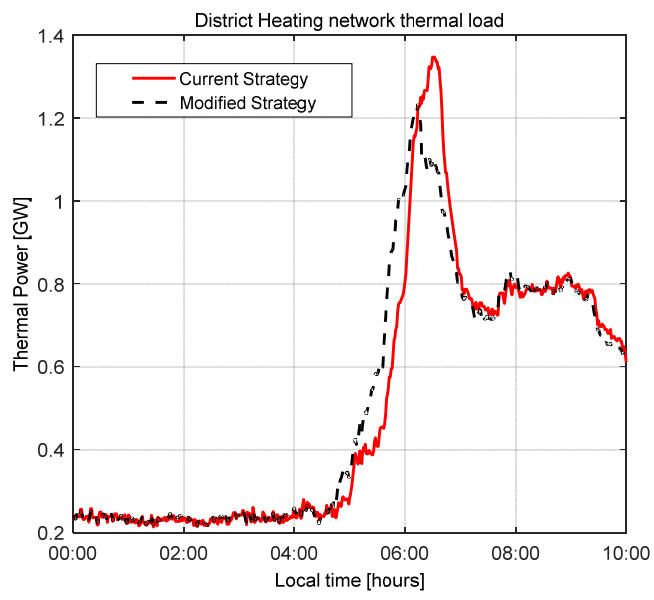

Figure 5: Thermal loads in a typical winter day obtained by using the current request profiles and the modified request profiles [26].

As shown in Fig. 4, the Pollution scenario in DHN starts with a first simulation of thermal loads in DHN executed by the District Heating Simulator (DHS) module as depicted in [26] (step 1 in Fig. 4). On the one hand, DHS retrieves the needed information from GIS, SIM and weather data, which are data-sources integrated through Data Integration Platform (steps 2-4 in Fig. 4). On the other hand, DHS exploits a Smart Metering Architecture to access i) historical data collected by IoT devices deployed in buildings and ii) information about energy consumption and production of the power plant, i.e. Distributed Generation module (steps 5-6 in Fig. 4). In buildings, IoT devices monitors i) indoor air temperature in rooms 
and ii) mass flowrate and water temperature in the heat-exchanger. The result of DHS simulation is the new thermal request of buildings and the thermal load of the plant, as shown in Fig. 5 [26]. Finally, to evaluate the pollution scenario with the new DHS strategy, results of DHS simulation are given as input to DIDEM module (step 8 in Fig. 4). Then, DIDEM retrieves the other inputs about GIS, weather, census and thermal energy production (steps 9-12 in Fig. 4). The final output of the whole Pollution scenario in DHN (step 13 in Fig. 4) is an estimation of pollution reduction with the new DSM strategy.

In the scope of this research activity, the implementation of the DIDEM model into the MESsi platform represent a first stage, as well as a benchmark towards the development of EC-LAB ESM platform. Its application is expected to bring significant advantages to Turin's energy planning, both for the energy and the air quality sector. In fact, on the one hand, the simulation of energy systems will be completed with information on the associated environmental impacts. On the other hand, the changes of air quality given by a selected scenario will be based on a complex online energy model. Shifting the structure of DIDEM from an offline to an online (web-based) conception will then bring significant advantages for its application, as the communication and information structures will enable a continuously updated data exchange between the modules.

\section{CONCLUSION}

The implementation process is expected to lead to an updated and more integrated version of MESsi platform. This is expected to bring significant advantages in MES modelling, both in support of energy and environmental planning. In fact, if the energy aspects are considered, the resulting energy balances from the evaluated scenarios are integrated with important information on the related impacts on human health and the environment. Conversely, if the environmental aspects are considered, a multi-layered and online-based simulation of the entire energy system would allow a precise and complete characterization of the emission sources, lowering the overall uncertainty of the process. This represents a significant contribution to the development of these kind of models, since the main challenge in modelling health effects and costs of air pollution is the quantification of the overall uncertainty associated to the assessment chain. The main challenges in the applicability of DIDEM is given by its extended usability. An advanced dispersion model like CALPUFF, in fact, requires detailed weather and geophysical datasets (usually only available upon request to Local Environmental Agencies) and a high expertise of the user.

Indeed, the combined analysis of MES and environmental impacts could be applied to a great number of scenarios in urban areas. Examples of such scenarios, that interest the energetic behaviour of buildings, districts and cities are: i) Installation of Renewable Energy Sources, ii) Grid reconfiguration, iii) Demand Response and iv) Demand Side Management.

The particular modular structure of MESsi makes it suitable to be continuously developed and integrated with new components. In this perspective, a brief presentation of the research activity at the Energy Center Lab of Turin Polytechnic has been reported. EC-LAB is currently working on development of an energy scenario making (ESM) modelling platform, able to simulate complex energy systems and analyse different management schemes. The MESsi model constitutes the basic framework of this research project. It is expected to be integrated with an increasing number of existing (and upcoming) modelling tools and tested on the urban energy system of Turin town in Italy.

Among the all potential application of such an extended modelling platform, the study of urban mobility networks could also be a topic of interest, as lowering energy consumption and emissions from traffic still represents one of the main challenges. Regarding the environmental aspects, future research activity will also include the implementation of the 
calculation of global-scale impacts in MESsi, through the development of a module for greenhouse gas accounting and balancing.

\section{REFERENCES}

[1] Mancarella, P., MES (multi-energy systems): An overview of concepts and evaluation models. Energy, 65, pp. 1-17, 2014.

[2] Chicco, G. \& Mancarella, P., Distributed multi-generation: a comprehensive view. Renew Sustain Energy Rev., 13(3), pp. 535-551, 2009.

[3] Molitor, C., Gross, S., Zeitz, J. \& Monti, A., Mescosa multienergy system co-simulator for city district energy systems. IEEE Transactions on Industrial Informatics, 10(4), pp. 2247-2256, 2014.

[4] Allegrini, J., Orehounig, K., Mavromatidis, G., Ruesch, F., Dorer, V. \& Evins, R.A., Review of modelling approaches and tools for the simulation of district-scale energy systems. Renewable and Sustainable Energy Reviews, 52, pp. 1391-1404, 2015.

[5] Keirstead, J., Jennings, M. \& Sivakumar, A., A review of urban energy system models: Approaches, challenges and opportunities. Renewable and Sustainable Energy Reviews, 16(6), pp. 3847-3866, 2012.

[6] Van Beuzekom, I., Gibescu, M. \& Slootweg, J., A review of multi-energy system planning and optimization tools for sustainable urban development. PowerTech, 2015 IEEE Eindhoven, pp. 1-7, IEEE, 2015.

[7] Kampa, M. \& Castanas, E., Human health effects of air pollution. Environ. Pollut. 151, pp. 362-367, 2008.

[8] Kirtman, B., Near-term Climate Change: Projections and Predictability, eds G.K. Plattner, M. Tignor, S.K. Allen \& J. Boschung, 2013.

[9] Panepinto, D., Brizio, E. \& Genon, G., Atmospheric pollutants and air quality effects: limitation costs and environmental advantages (a cost-benefit approach). Clean. Techn Environ. Policy, 16, pp. 1805-1813, 2014.

[10] University of Stuttgart, Institute of Energy Economics and the Rational Use of Energy (IER), ExternE - Extern. Costs Energy. http://www.externe.info/. Accessed on: 26 Mar. 2018.

[11] European Commission, ExternE - Externalities of Energy: Methodology 2005 Update, Office for Official Publication of the European Communities, Luxembourg Eurostat, Harmonized Indices of Consumer Prices (HICP) - Main Tables, 2005. http://ec.europa.eu/eurostat/web/hicp/data/main-tables. Accessed on: 26 Mar. 2018.

[12] van der Kamp, J. \& Bachmann, T.M., Health-related external cost assessment in Europe: methodological developments from ExternE to the 2013 Clean Air Policy Package. Environ. Sci. Technol., 49, pp. 2929-2938, 2015.

[13] Henschel, S. et al., Air Pollution Interventions and Their Impact on Public Health, 57(5), pp. 757-768, 2012

[14] Bottaccioli, L., Patti, E., Macii, E. \& Acquaviva, A., Distributed infrastructure for multi-energy-systems modelling and co-simulation in Urban Districts. Proceedings of the 7th International Conference on Smart Cities and Green ICT Systems, pp. 262269, 2018.

[15] Pau, M. et al., A cloud-based smart metering infrastructure for distribution grid services and automation. Sustainable Energy, Grids and Networks, 2017.

[16] Ravina, M., Panepinto, D. \& Zanetti, M.C., DIDEM - An integrated model for comparative health damage costs calculation of air pollution. Atmospheric Environment, 173, pp. 81-95, 2018. 
[17] WHO, Health Risks of Air Pollution in Europe - HRAPIE Project. Recommendations for concentration-response functions for cost-benefit analysis of particulate matter, ozone and nitrogen dioxide, World Health Organization, Regional Office for Europe: Copenaghen, Denmark, p. 54, 2013.

[18] WHO, Review of evidence on health aspects of air pollution - REVIHAAP Project. Technical report, The WHO European Centre for Environment and Health: Bonn, Germany, p. 302, 2013.

[19] U.S. Environmental Protection Agency (US EPA), CALPUFF modeling system user's manual, version 6, 2011.

[20] QGIS open source software. https://www.qgis.org/it/site/. Accessed on: 3 Mar. 2018.

[21] Golden Software. Surfer ${ }^{\circledR}$. http://www.goldensoftware.com/products/surfer. Accessed on: 26 Mar. 2018.

[22] Ravina, M., Panepinto, D. \& Zanetti, M.C., District heating system: evaluation of environmental and economic aspects. J. Environ. Impacts, 1(4), pp. 420-432, 2018. DOI: $10.2495 /$ EI-V1-N4-420-432.

[23] Ravina, M., Panepinto, D., Zanetti, M.C. \& Genon, G., Environmental analysis of a potential district heating network powered by a large-scale cogeneration plant. Environmental Science and Pollution Research, 24, pp. 13424-13436, 2017. DOI: 10.1007/s11356-017-8863-2.

[24] Bottaccioli, L., Patti, E., Macii, E. \& Acquaviva, A., GIS-based software infrastructure to model PV generation in fine-grained spatio-temporal domain. IEEE Systems Journal, 2017. DOI: 10.1109/JSYST.2017.2726350.

[25] Bottaccioli, L., Estebsari, A., Patti, E., Pons, E., \& Acquaviva, A., A novel integrated real-time simulation platform for assessing photovoltaic penetration impacts in smart grids. Energy Procedia, 111, pp. 780-789, 2017. DOI: 10.1016/j.egypro.2017.03.240.

[26] Brundu, F.G. et al., Iot software infrastructure for energy management and simulation in smart cities. IEEE Transactions on Industrial Informatics, 13(2), pp. 832-840, 2017. DOI: 10.1109/TII.2016.2627479.

[27] Bottaccioli, L. et al., A flexible distributed infrastructure for real-time cosimulations in smart grids. IEEE Transactions on Industrial Informatics, 13(6), pp. 3265-3274, 2017. DOI: 10.1109/TII.2017.2702206.

[28] Bottaccioli, L. et al., Building energy modelling and monitoring by integration of IoT devices and Building Information Models. Computer Software and Applications Conference (COMPSAC), 2017 IEEE 41st Annual, 1, pp. 914-922, IEEE, 2017. DOI: 10.1109/COMPSAC.2017.75.

[29] Borchiellini, R., The Energy Center Initiative at Politecnico di Torino: Practical experiences on energy efficiency measures in the municipality of Torino. International Journal of Heat and Technology, 35(1), Sept. 2017. DOI: 0.18280/ijht.35Sp01XX. 